\title{
Innovative mixed reality approach to rock mass mapping in underground mining
}

\author{
IE Onsel SRK Consulting, Canada
}

D Stead Simon Fraser University, Canada

W Barnett SRK Consulting, Canada

\author{
L Zorzi SRK Consulting, Canada
}

A Shaban SRK Consulting, Canada

\begin{abstract}
Traditional methods of mapping in mines use a geological compass for orientation measurements, a tape measure for length measurements and pen/paper for sketching and taking notes. This has been improved in recent years with a change from compass to smart phones/tablets and the use of tablets for sketching photographic images. A significant improvement is the use of remote sensing e.g. LiDAR and photogrammetry to collect data with subsequent office-based data processing. However, the collected $3 D$ data is usually displayed on 2D monitors which is a very different experience in comparison to fieldwork. The perception of 3D geometry and scale are lost, which may result in biased and limited interpretations. The Microsoft HoloLens is an augmented reality headset comprising a computer with a transparent screen and a 3D scanner. This device allows the creation of a 3D map of the real-world almost in real-time. Using this map, it becomes possible to place virtual objects within the real-world and to know the location of the HoloLens user. As the screen is transparent HoloLens users can see the real-world along with virtual objects, such as previous mapping and drilling completed anywhere within the underground mining operation. Advantage of state-of-the-art new VR/MR technology was taken and a Microsoft HoloLens application which can be used to annotate/draw directly on a rock face was developed and the necessary spatial data in the field for use during mapping is made available. After field work has been undertaken, the collected data can be easily exported to other software without further post-processing. Holographic mapping procedures for virtual rock outcrops constructed from remote sensing data, e.g. ground and UAV based LiDAR and photogrammetry was also developed. This allows office-based mapping of virtual outcrops. The developed software will improve direct outcrop mapping and office-based mapping using remote sensing data.
\end{abstract}

\section{Introduction}

In recent years, there has been a dramatic increase in the use of mixed and virtuality reality, MR/VR, techniques in mining engineering. The majority of these applications have focussed on improving visualisation of engineering projects including open pits and surface mine reclamation. Trimble (2016) developed an application where holographic open pits placed on boardroom tables allow communication between mine engineers in a mine production and truck haulage capacity. BGC Engineering used MR/ VR and holographic images to communicate the life stages of an Alberta oil sands mine emphasizing the environmental reclamation of the site in a unique communication format (Loook 2017). MineLife, similarly is a VR software for mine site planning developed by Llamazoo (2018). At Simon Fraser University, the Engineering Geology and Resource Geotechnics Research Group has used MR technology on numerous engineering projects including landslides and open pits (Onsel et al. 2018) (Figure 1). 

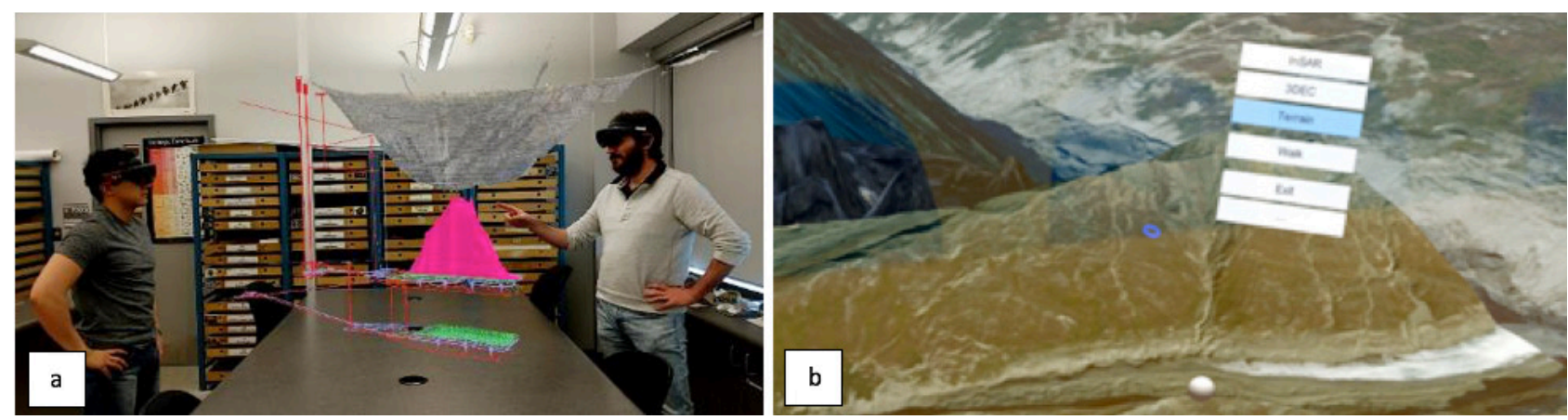

Figure 1 Example application of MR/VR for the geovisualization of engineered and natural slopes. a: Palabora open pit and block cave mine, South Africa b: Fels landslide, Alaska (Onsel et al. 2019)

In this paper, a MR/VR application for geotechnical site characterization using the Microsoft HoloLens 2 and virtual reality headsets is demonstrated. A HoloLens comprises a short distance 3D scanner that can be used on site for data collection and mapping. It is also capable of visualizing 3D multi-sensor remote sensing data, both in the field and at the office. Virtual reality headsets provide an immersive experience for users where they can work with larger 3D multi-sensor remote sensing data.

\section{$2 \quad$ Mixed and virtual reality}

In virtual reality users are immersed in a fully artificial digital environment. In augmented reality, virtual objects are overlain on the real world whereas in augmented virtuality real objects are overlain on the virtual world. Mixed reality (Figure 2) refers to a continuum that encompasses both augmented reality and augmented virtuality via immersive technology (Milgram et al. 1994).

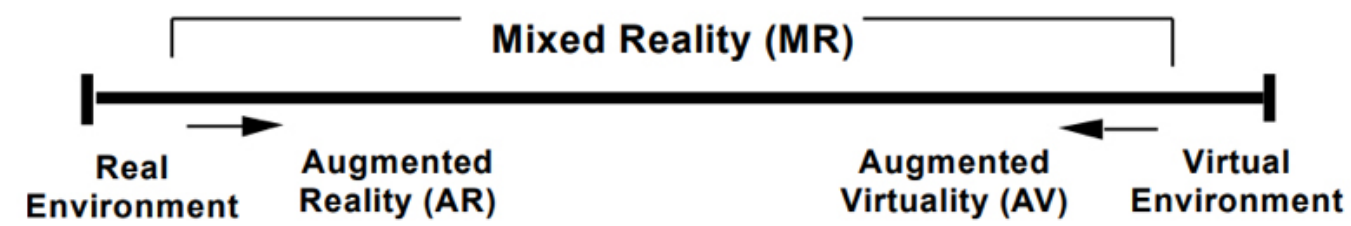

Figure 2 Reality-virtuality continuum (modified after Milgram et al. 1994)

The Microsoft HoloLens 2 (Figure 3a) is a mixed reality headset with transparent holographic lenses that allow users to see the real world. It has a 1-MP time-of-flight depth sensor and holographic processing unit for creating a real-time environment mesh which can be used as a map to place virtual objects into the real world. The unit is untethered having an internal computer which is powered by Qualcomm snapdragon 850 processor with 4GB memory and uses Windows Holographic operating system to run Universal Windows Platform (UWP) applications. UWP applications for the HoloLens are created by using Unity game engine software and the Mixed Reality Toolkit, an open source project supported by Microsoft that provides primary scripts in order to accelerate the development of mixed reality applications.

Windows Mixed Reality Headsets (WMRH) are virtual reality headsets tethered to computers with Windows 10 operating system. They are not capable of showing the real world and creating a map of it, however, they provide an excellent immersive experience for users. Depending on the computer processing power much larger virtual outcrops with multi-sensor remote sensing data can be displayed. The UWP applications developed for HoloLens are compatible to WMRH's. One of the latest WMRH is the HP Reverb (Figure 3b) which has displays with 9.3 megapixels and 114-degree field of view providing sharp, clean images. 

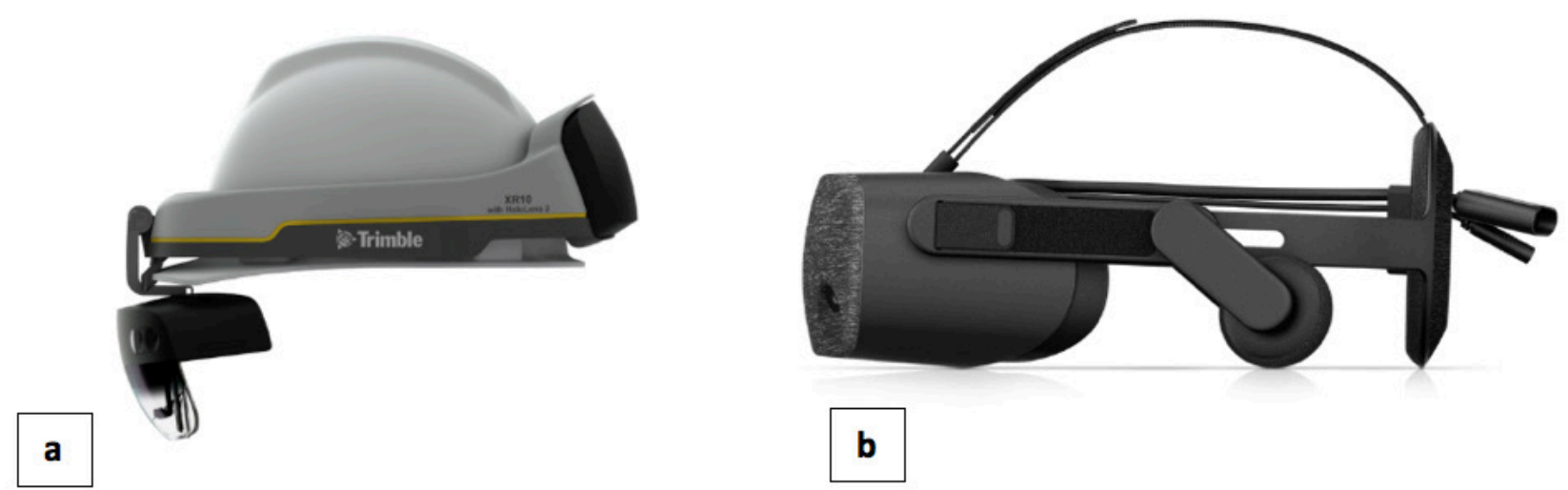

Figure 3 a) Trimble XR10 with Microsoft HoloLens 2 mounted on safety helmet (Microsoft, 2019), b) HP Reverb Windows Mixed Reality Headset (HP 2019)

\section{EasyMineXR MR/VR}

EasyMineXR MR/VR is a mixed and virtual reality application developed under a joint Mitacs Accelerate project between SRK Consulting and Simon Fraser University for mapping outcrops on site and virtual outcrops in the office. The software runs on both the HoloLens and on computers with virtual reality headsets.

\subsection{Outcrop mapping in the field}

\subsubsection{Scanning and georeferencing}

EasyMineXR MR/VR takes advantage of the built-in 3D scanner in the HoloLens to collect data on site. When the HoloLens user looks directly toward an object at a distance between $0.8 \mathrm{~m}$ and $3 \mathrm{~m}$, the HoloLens scanner creates a mesh of the object with a resolution of ca. $5 \mathrm{~cm}$. Therefore, the EasyMineXR user simply needs to walk along, and view the investigated outcrops to create a mesh in quasi-real time. Users have the option to view, hide, and colorize the mesh, Figure 4. After finishing the scan, the user may visualize a small-scale preview of the mesh, before saving the 3D dataset.

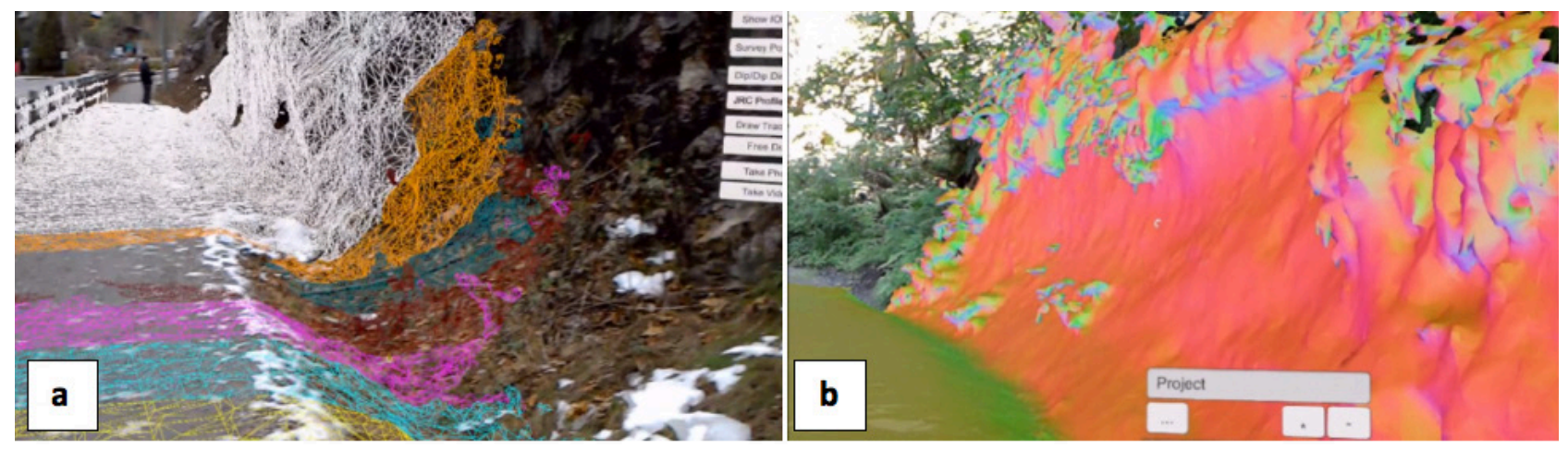

Figure 4 a) Mesh of the outcrop created by HoloLens, b) mesh coloured according to surface orientations

Georeferencing is performed using a compass (to set the north in the application) and a survey point located on the outcrop (to convert the local HoloLens coordinate system to a project or real-world system). Alternatively, two survey points may be used.

\subsubsection{Importing data}

Once the registration process is complete, additional georeferenced data can be imported into EasyMineXR MR/VR. For example, meshes and drillhole data exported from Leapfrog Geo (Leapfrog 2016) will appear in their real-world coordinates, allowing the user to visualize data collected at a different time, providing 
the opportunity for more sophisticated on-site interpretation (Figure 5). Imported objects may be visualized on the map and users can see their position inside the mine.

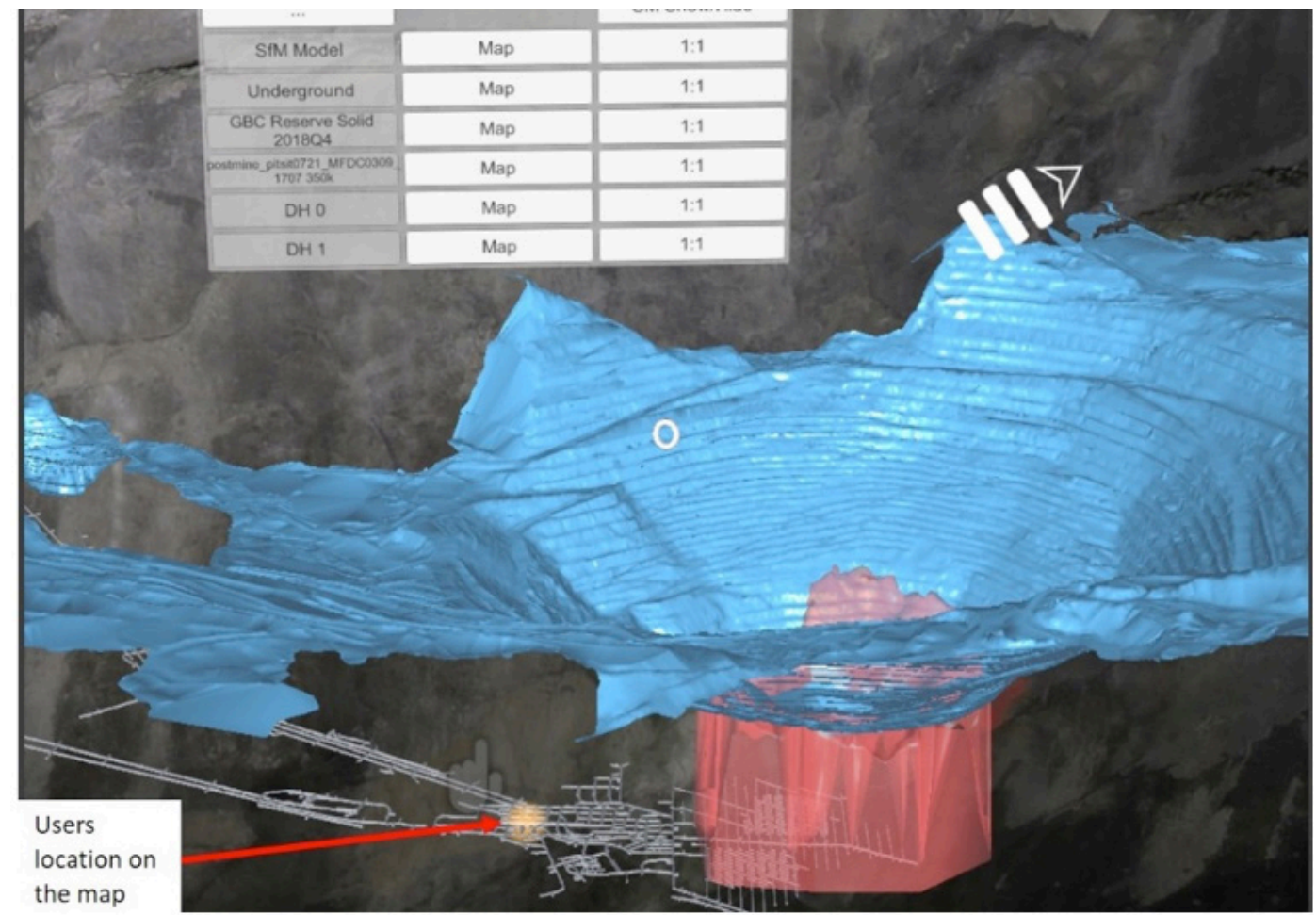

Figure 5 Examples of 3D objects imported in EasyMineXR MR/VR

\subsubsection{Orientation measurement}

An orientation measurement tool was developed to determine the dip and dip direction of joint surfaces (Figure 4). When the user points toward the joint surfaces, EasyMineXR instantaneously computes the dip and dip direction, based on the orientation of the mesh. The user is then able to save the orientation data and display it in real time on a stereonet. This tool significantly speeds up geomechanical mapping, as there is no need to read the orientation data from the compass (or smartphone). It also improves the safety of the user, as it allows the structure to be mapped without physically accessing the rock face. Finally, the user can run the Fuzzy K-Mean algorithm (Hammah \& Curran 1999) to estimate the number and average orientation of the joint sets.

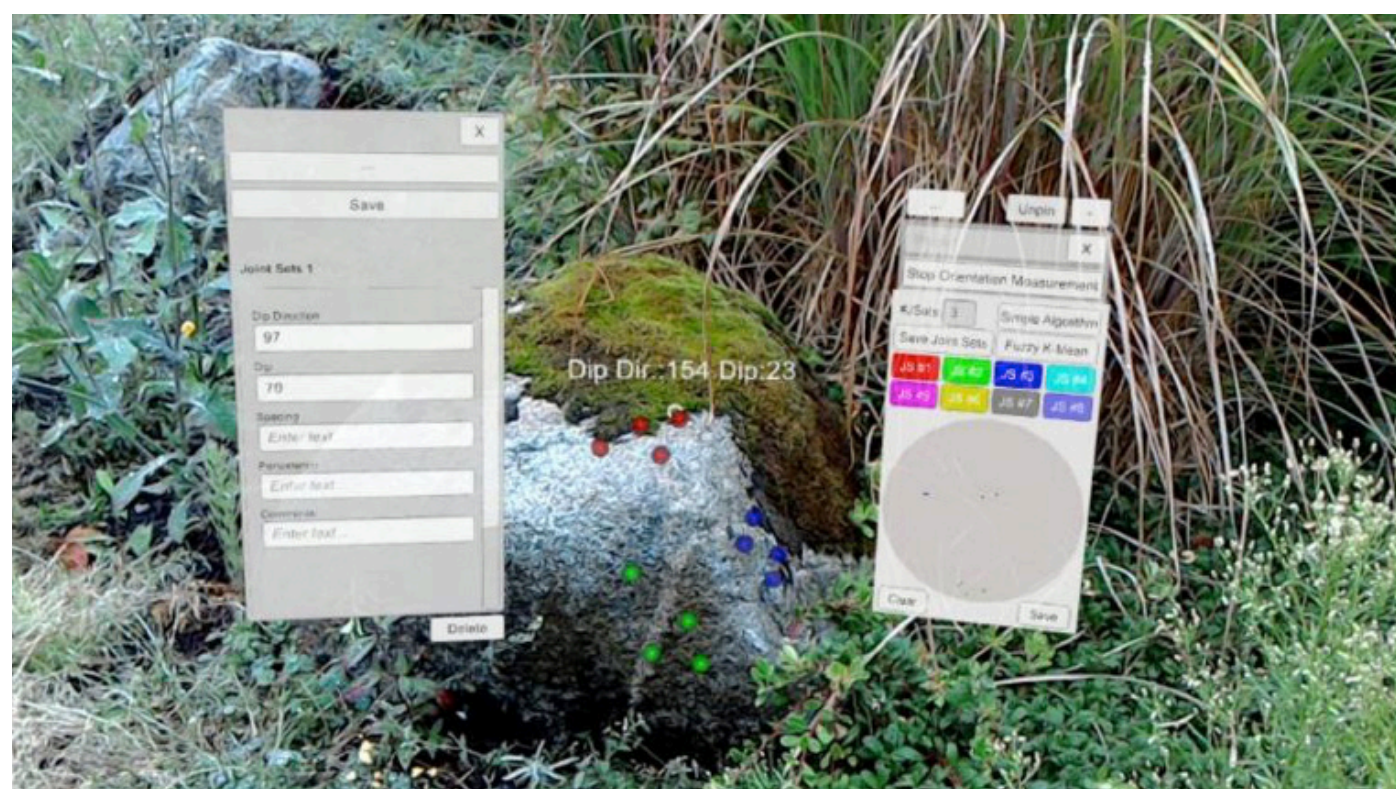

Figure 6 The use of EasyMineXR MR for joint surface orientation measurement 


\subsubsection{Tracing}

Users can perform HoloLens geological, structural, and geomechanical mapping by drawing traces along the observed features (Figure 7). The user can define the feature type, before the trace is saved as a 3D polyline. The shape can also be annotated using a form that is visualized by selecting the 3D polyline. Different form templates can be used, depending on the structure type (e.g., fault, joint, lithological contact, etc.). The 3D polylines can be exported as comma separated values (csv) files. Georeferencing data is maintained during this process, eliminating the need for any post-processing.

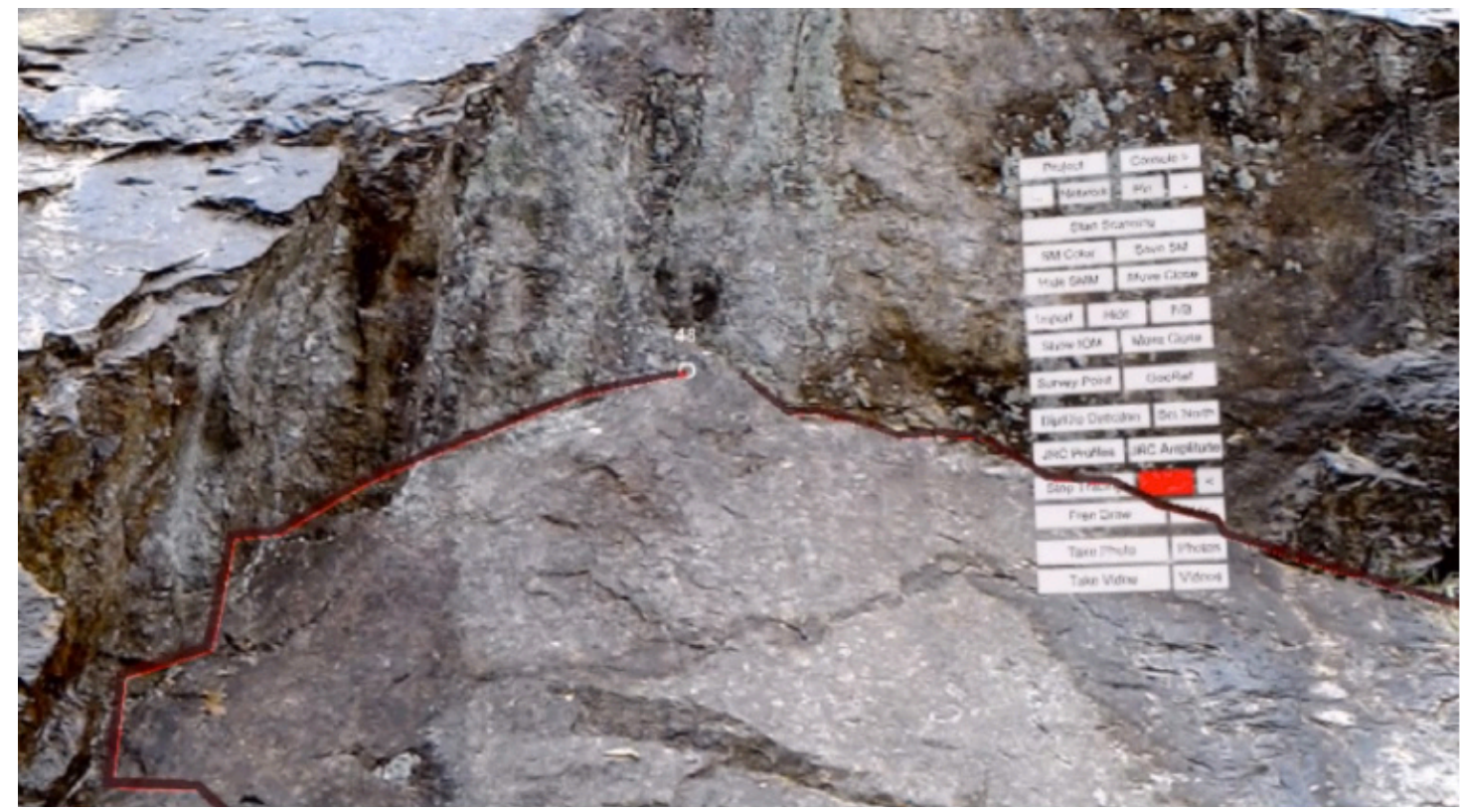

Figure 7 Trace drawn onto the outcrop in EasyMineXR MR

\subsubsection{EasyMineXR MR/VR tools}

A Ruler tool is used to measure the length of structures (Figure 8). EasyMineXR MR/VR also displays charts such as joint roughness profiles from Barton and Choubey (1977) (Figure 9).

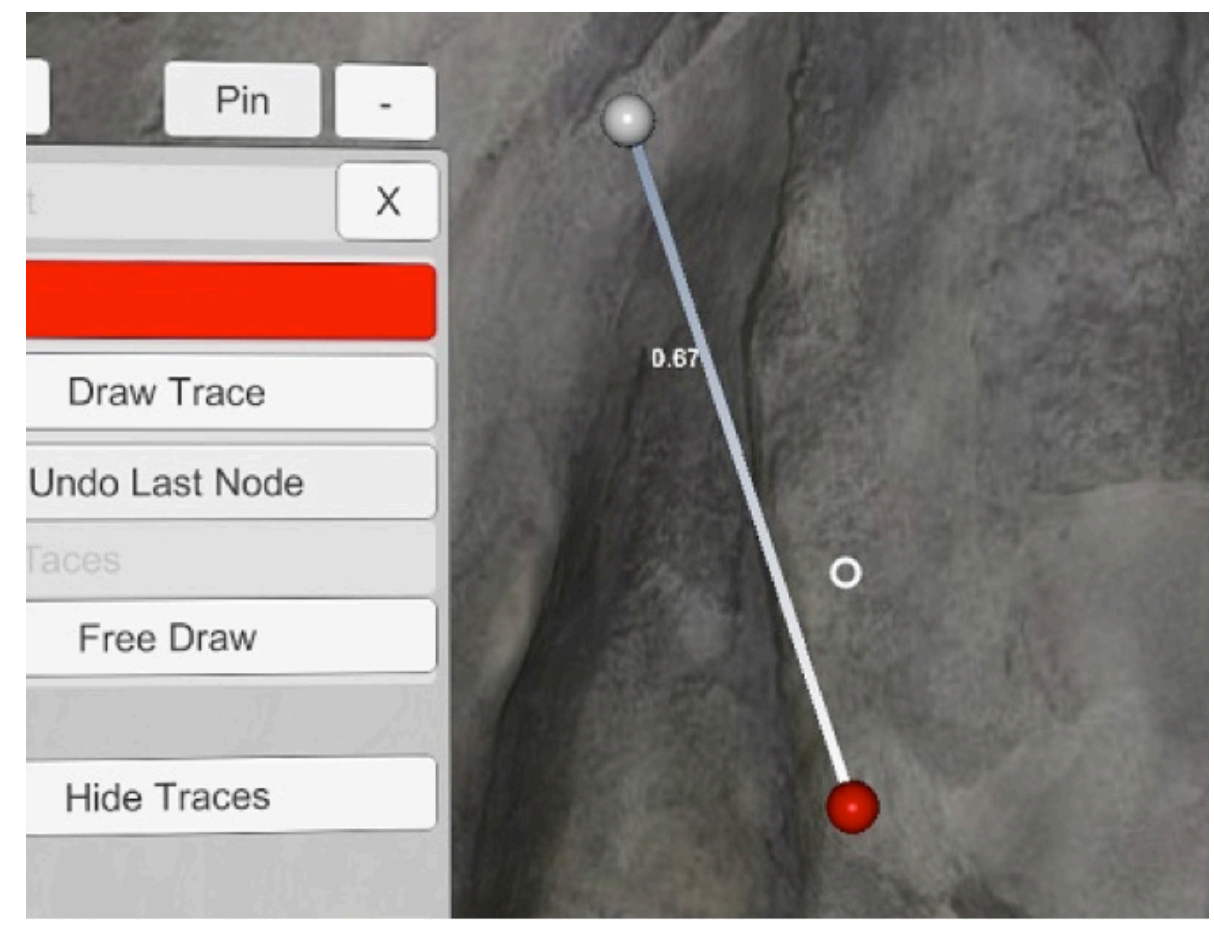

Figure 8 3D measurements performed using the EasyMineXR MR/VR ruler tool 


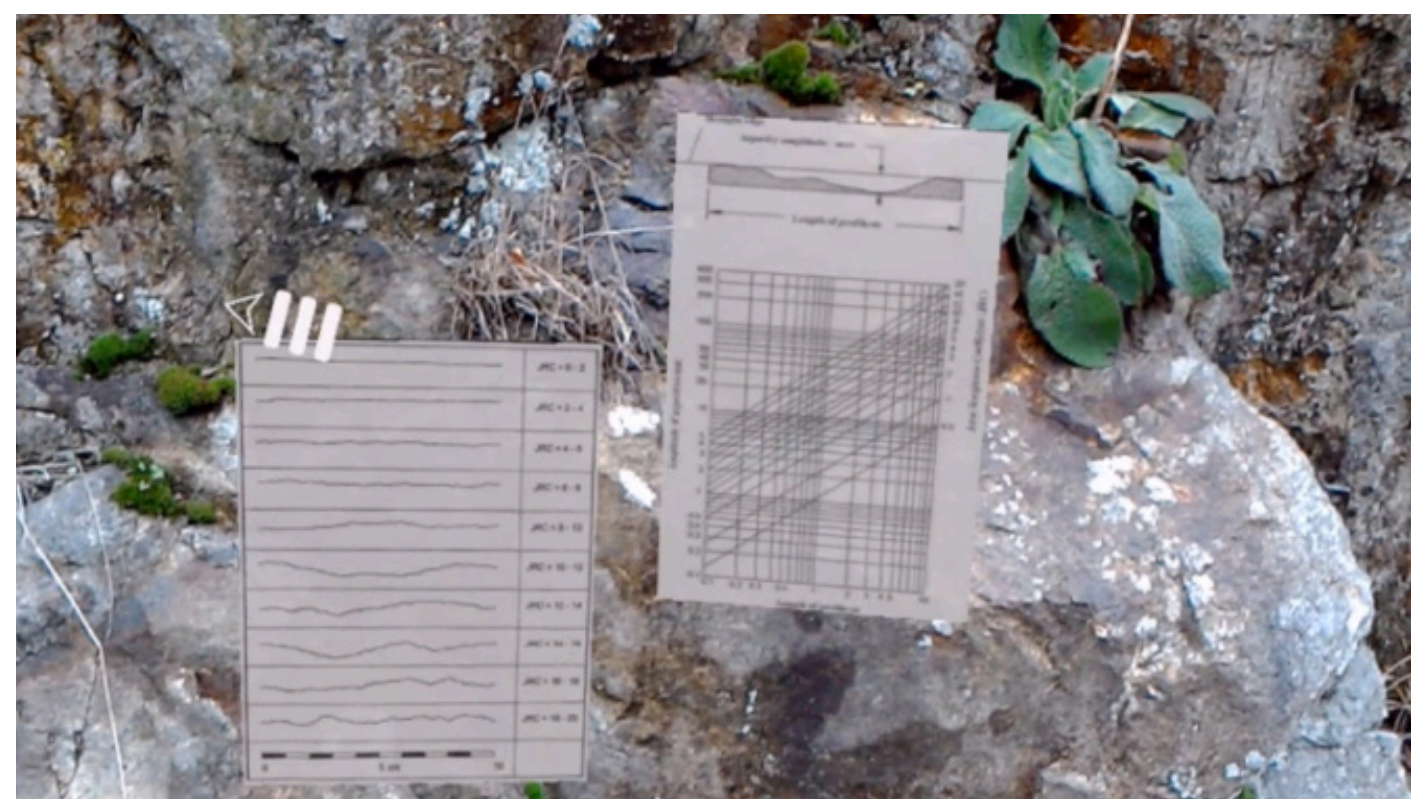

Figure 9 Joint roughness charts visualized in EasyMineXR MR

\subsection{Virtual outcrop mapping}

Virtual outcrops are created by using remote sensing methods. Photogrammetry and laser scanners acquires geometry with visible colours of the outcrop as point clouds. Data on signal return intensity (represented using colour) can also collected by laser scanners. In addition, thermal and hyperspectral cameras provide additional data on rock masses. By combining these datasets, virtual outcrops can be created as mesh files which can be imported to EasyMineXR MR/VR.

The main difference between the use of EasyMineXR in MR and VR is the data size limit. Mesh files with more than 200k vertices frequently overtax the current processing power of the HoloLens. EasyMineXR in VR allows much higher data limits and data processing ability dependent on the computer and graphics card specifications. To map with the HoloLens users have to walk to access different parts of the outcrop and have the option of cleaning or preparing the outcrop for detailed observations. In VR, users can fly and teleport across an outcrop, but are limited by outcrop image resolution. In VR, georeferencing is not required as the software already uses real world coordinates.

Mapping procedures in VR are similar to those used in HoloLens field mapping. Data is collected by tracing and orientation measurements that are part of the EasyMineXR tool set. In addition, users can switch between remote sensing data and the original visual image allowing comparison and significantly improving the mapping product and interpretation.

\section{$4 \quad$ Field tests}

Initial field mapping tests using the HoloLens have been undertaken at rock slopes in West Vancouver and in Burnaby, BC. Tests for underground mapping were undertaken in the Othello Tunnels, near Hope, BC (Figure 10).

This was followed by underground mapping at Grasberg, Indonesia (Figure 11) and in Orapa open pit, Botswana. The HoloLens performed well under low light conditions and was not significantly affected by the humidity and dusty environment.

Another mine test was then undertaken at the Seabee Mine in Saskatchewan where bedrock structures were mapped, and the results exported into Leapfrog (Figure 12). It is observed that once familiar with the Hololens, mapping time is similar to mapping on paper. There are some significant benefits that save time. All data is immediately electronically available for importing into 3-D modelling software for further 
interpretation. Hololens also allows all previous mapping data to be visible in 3D while mapping, thereby enabling and arguably "forcing" the mapping geologist or engineer to interpret the observations in 3D at the rock face. No other technology creates a similar fundamental benefit to data collection in earth sciences.

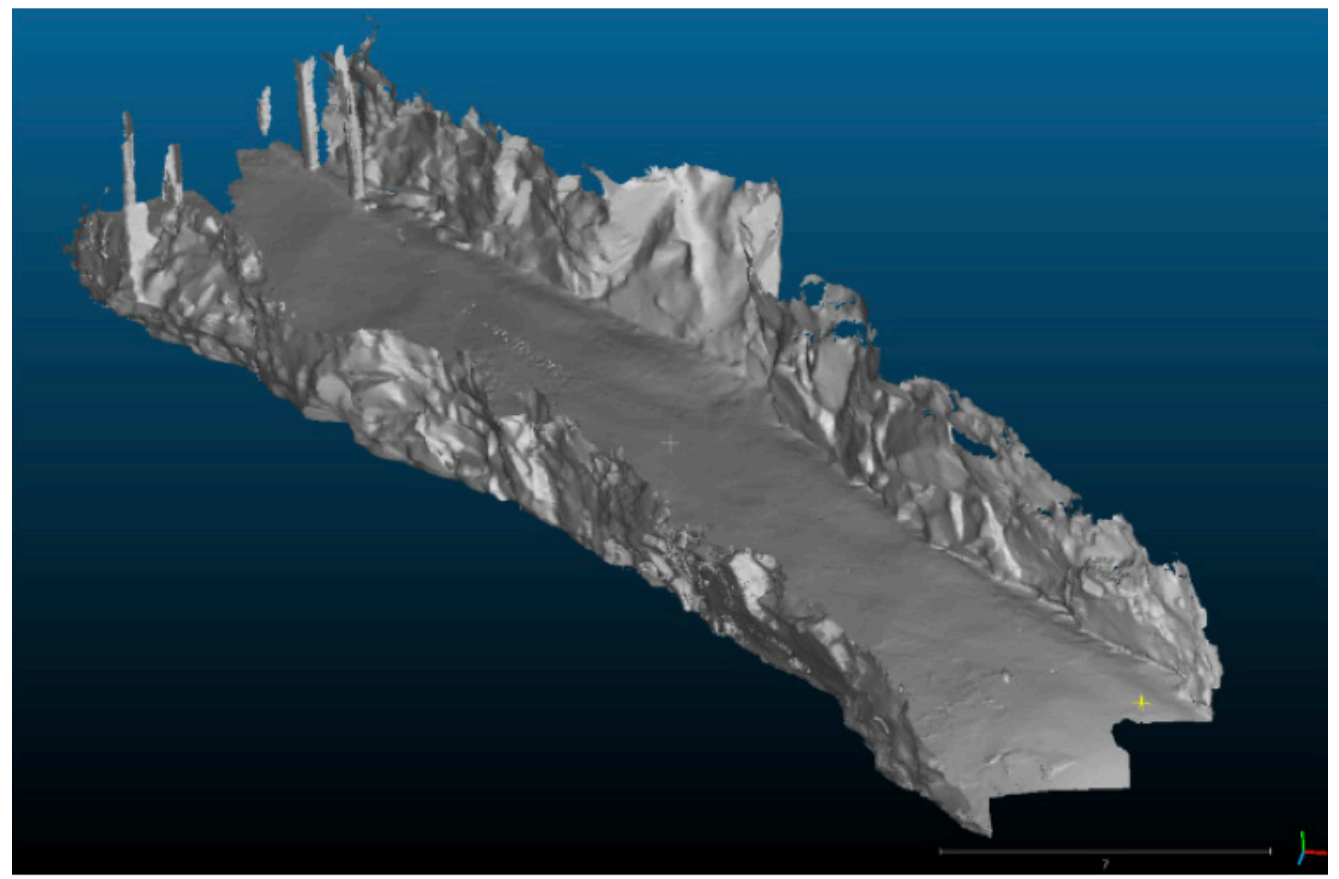

Figure 10 Othello Tunnel HoloLens scan result

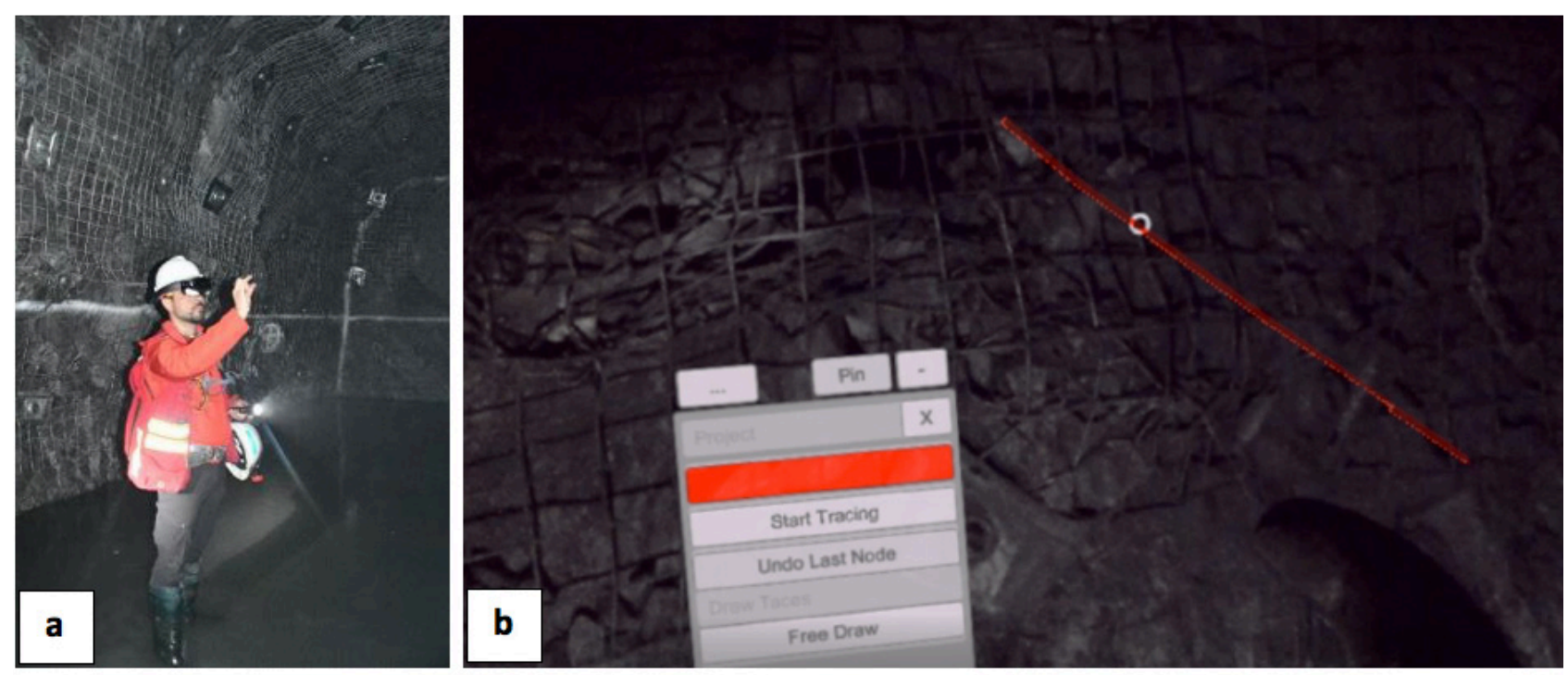

Figure 11 a) Grasberg mine underground test, b) user's point of view

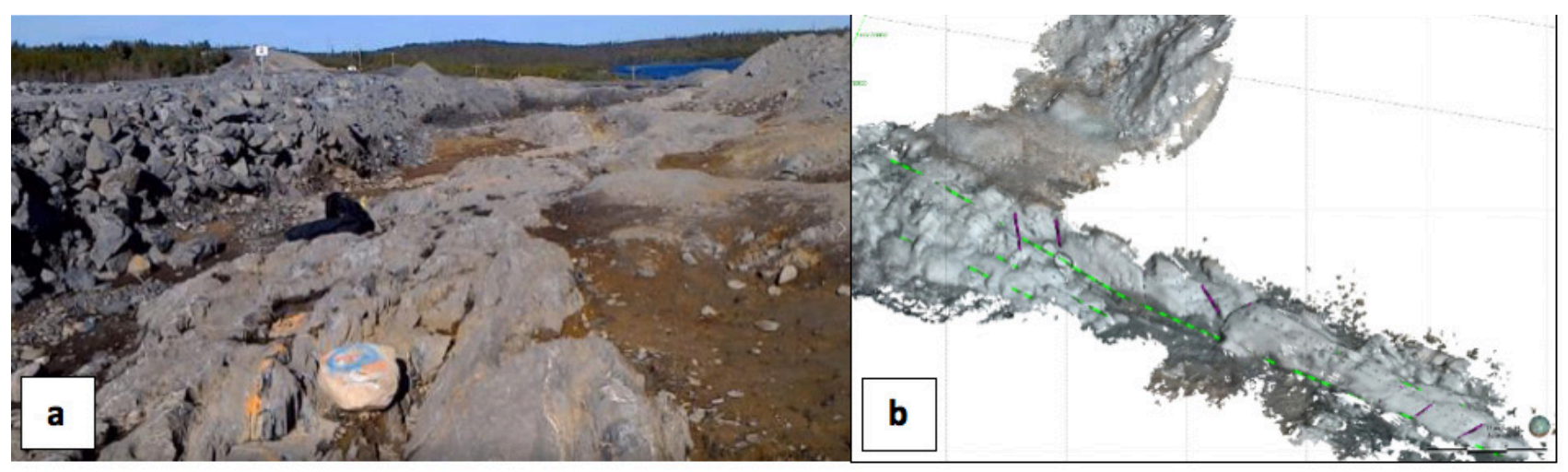

Figure 12 Seabee mine a) bedrock, b) exported data in Leapfrog 


\section{Conclusions}

This paper presents EasyMineXR MR/VR an innovative application for geotechnical site characterization using the Microsoft HoloLens 2 and virtual reality headsets, without removing the ability to be at the actual rock for data collection. The HoloLens is a MR headset with a transparent screen capable of displaying virtual objects in the real world and a 3D scanner for creating a 3D mesh map of the realworld environment. The HoloLens has shown to be suitable for both onsite data collection and mapping of outcrops. It was also demonstrated that Virtual Reality headsets provide an immersive experience for users where they can map virtual outcrops comprising 3D multi-sensor remote sensing data.

The future will bring a new generation of MR headsets with improved processing power and higher resolution scanners allowing collection of more detailed data such as joint roughness. It will be possible to perform advanced data processing on site such as running numerical models in real time, which will completely change the workflow of rock mass characterization where geologists and engineers collect data in the field and post process it in the office. MR and VR headsets will also become lighter, smaller and cheaper which will transform them into a common used device like a smart phone. Geologist and engineers should take advantage of this and improve their field, office work, training and communication by developing and using MR/VR software.

\section{References}

Barton, N, \& Choubey, V 1977, 'The shear strength of rock joints in theory and practice', Rock Mechanics, Vol. 10, no. 1-2, pp. $1-54$.

Hammah, RE \& Curran, JH 1999, 'On distance measures for the fuzzy K-means algorithm for joint data', Rock Mechanics and Rock Engineering, vol. 32, no. 1, pp. 1- 27.

HP 2019, HP Reverb Virtual Reality Headset - Pro Edition. Available from: <https://www8.hp.com/us/en/vr/reverb-vr-headset. html>. [1 April 2020].

Leapfrog 2016, Leapfrog Geo, version 4.0.

LLamazoo 2018, MineLife VR - Virtual Reality Mine Site Planning | LlamaZOO Interactive. Available from: <https://youtu.be/ wGHmlQwlefY>. [1 April 2020].

Loook 2017, BGC Engineering and LOOOK: Holographic Mine Reclamation. Available from: <https://youtu.be/-WYJfbktnl8>. [1 April 2020].

Microsoft 2019, Trimble XR10 with HoloLens 2. Available from: <https://news.microsoft.com/microsoft-at-mwc19/photos/ trimble-xr10-with-hololens-2/>. [1 April 2020].

Milgram, P, Takemura, H, Utsumi, A \& Kishino, F 1994, 'Augmented Reality: A class of displays on the reality-virtuality continuum', in Proceedings of Telemanipulator and Telepresence Technologies, pp. 2351-34.

Onsel, IE, Chang, O, Mysiorek, J, Donati, D, Stead, D, Barnett, W \& Zorzi, L 2019, 'Applications of mixed and virtual reality techniques in site characterization', in 26th Vancouver Geotechnical Society Symposium, Vancouver.

Onsel, IE, Donati, D, Stead, D \& Chang, O 2018, 'Applications of virtual and mixed reality in rock engineering', in 52nd US Rock Mechanics/Geomechanics Symposium, Seattle.

Trimble 2016, Trimble Connected Mine. Available from: < https://mixedreality.trimble.com/connected-mine/>. [1 April 2020]. 
